\title{
Towards Improving the Performance of Creighton Mine's Natural Heat Exchange Area
}

\author{
Negar Saeidi ${ }^{1}$, Lorrie Fava ${ }^{1}$, Cheryl Allen ${ }^{2}$ \\ ${ }^{1}$ MIRARCO - Mining Innovation \\ 935 Ramsey Lake Rd, Sudbury, ON, Canada \\ nsaeidi@mirarco.org; lfava@mirarco.org \\ ${ }^{2}$ Vale Canada Limited, Ontario Operations \\ 18 Rink St, Copper Cliff, ON, Canada \\ cheryl.allen@vale.com
}

\begin{abstract}
The Canadian climate features long, cold winters and short, mild summers that provides the opportunity for Vale's Creighton Mine in Sudbury, ON, Canada to manage mine cooling requirements over a 50 year period. The intake air for Creighton Mine's ventilation system is conditioned by continuously pulling ambient air through the Natural Heat Exchange Area (NHEA). The NHEA is a large-scale volume created by previous open-pit and block cave mining at Creighton that contains significant broken rock tonnage with the ability to store ambient coolth and heat on a seasonal basis.

The study reported in this paper is part of an on-going collaborative research project conducted by Vale and MIRARCO. The project is investigating options to extend the efficiency of the NHEA system and provide cooler air to deeper levels of Creighton Mine. A threedimensional computational fluid dynamics (CFD) model is being developed and calibrated for dynamic modeling and simulation of the NHEA's thermal physics. The calibrated model will be used to support the assessment of both operation and system design variations to improve the NHEA's performance.
\end{abstract}

Keywords: Natural Heat Exchange Area (NHEA), Computational Fluid Dynamics (CFD) simulation, Creighton Mine, Underground Mine Ventilation

\section{Introduction}

The Canadian climate has allowed Creighton Mine to manage mine cooling requirements by exploiting an environmentally benign option, and eliminating fuel consumption for ventilation purposes, using the Natural Heat Exchange Area (NHEA) [1].

Creighton Mine, the deepest nickel mine in the world, is currently producing at a depth of approximately $2.5 \mathrm{~km}$, and is projected to continue production well into the future. Auto-compression of the downcast ventilating air increases with depth, and is the largest component of mine heat load in Canadian mines [2]. Normally, the air temperature increases $1^{\circ} \mathrm{C}$ with a $100 \mathrm{~m}$ increase in depth and therefore increases the required cooling capacity requirement as Creighton Mine develops deeper.

The current NHEA system has been managed over the years to meet the needs of the mine. Previous estimates from Creighton Mine have shown that the cooling capacity of the NHEA as it has been operated in the past will not be sufficient to continue mining without additional cooling that can be supplied using refrigeration plants [2]. Current efforts aim to postpone the capital cost of refrigeration plants by assessing modifications to the NHEA system or its current operation. Computational fluid dynamics (CFD) modeling and analysis of the NHEA is one of the approaches taken to study the NHEA system and subsequently improve its performance and provide additional cooling for the deeper levels of Creighton Mine.

This paper provides a concise introduction of the physics and dynamic thermal behaviour of the NHEA at Creighton Mine and a detailed description of the CFD model of the NHEA system. Primary guidance for potential engineering designs to improve the NHEA system are proposed based on the CFD simulations and historical data recorded at the NHEA. A brief explanation of the targets set for the next steps of this ongoing research work is also provided. 


\section{Physics and Performance of the Natural Heat Exchange Area (NHEA)}

The NHEA is the primary intake for the ventilation system of Creighton Mine and provides a natural source of air conditioning. The NHEA is a large-scale volume created during prior mining operations that contains significant broken rock tonnage. The NHEA is exposed to the environment at surface with an asymmetric concave face with a maximum depth of approximately $100 \mathrm{~m}$. The NHEA is connected to the underground ventilation network through 96 trench doors associated with boxholes and slusher trenches, as well as millholes and ore passes from the old mining. The accessible areas of the NHEA are divided into four partitions known as Blocks 1, 2, 5 and 6. Blocks 3 and 4 exist as well, but feature slusher trenches that are inaccessible. The plan view of the NHEA and the superimposed former mining area with the Block divisions are presented in Error! Reference source not found.. Approximately half of the trench doors are set closed and the remaining are set open on a year-round basis. The trench door states (closed or open) change depending on the associated season. Ambient air is continuously drawn through the broken rock with three main sets of fans underground, as illustrated in Error! Reference source not found.. The air pulled from the NHEA is directed through the open trench doors to a gathering area on the 800 Level (Error! Reference source not found..). Surveys have indicated a significant amount of air leakage in the NHEA system, which means that a significant amount of air is taking an unknown path to the gathering area ( 800 Level) and is not controlled through the trench door system. This will be discussed further in Section 4.

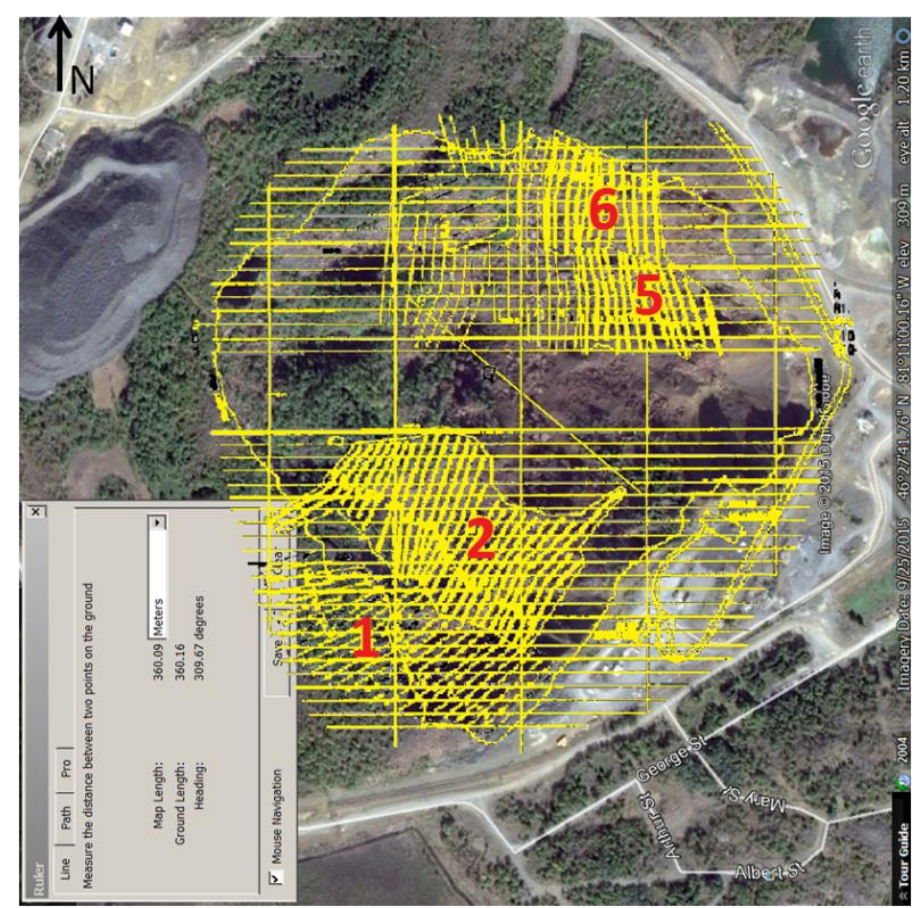

Fig. 1: The old workings divided into 4 groups known as Blocks 1, 2, 5 and 6 superimposed on the plane view of the NHEA [3]. 


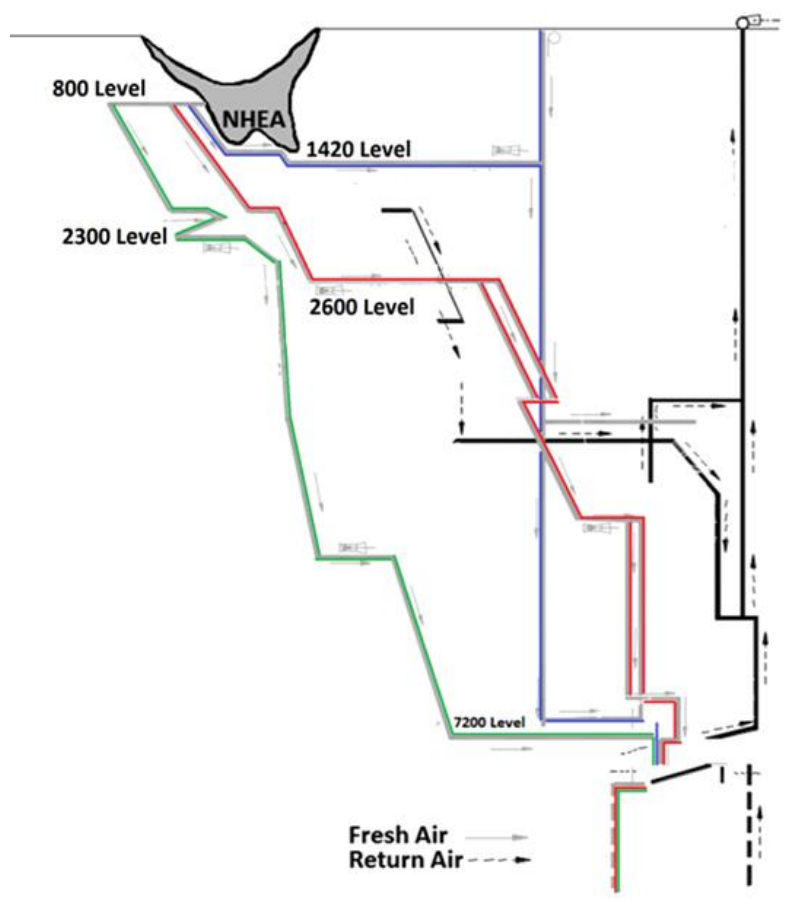

Fig. 2: Three main fans located at 1420, 2300 and 2600 Levels continuously pull air through NHEA and the gathering area on the 800 Level, to the three fresh air systems in green, blue and red [1].

Depending on the season, the ventilation air is heated or cooled as it is continuously drawn through the NHEA by the main fans. During winter, cold temperatures propagate from surface through the broken rock mass towards the open trench doors as a result of heat transfer between the incoming air and the broken rock that is initially warmer than the air. A similar process occurs in summer with a propagation of warm temperatures through the cooler broken rock mass. In other words, the NHEA is acting as a refrigeration system in summer and a heating system in winter.

Sensors are installed near the control doors to monitor the air temperature and the associated data is archived by a realtime data historian. The air temperature at a given open or closed trench door changes gradually as the result of heat transfer happening in the NHEA rock mass. Once the temperature at a given door surpasses a pre-established trigger value, the door state will be changed manually. The trigger temperature value at each trench door has been determined empirically and is a function of depth, block, and season.

Several uncontrolled factors, such as air leakage, ice formation in winter and flooding in summer, affect the efficiency of the NHEA's performance. This on-going study is aiming to enhance the knowledge of the physics of the NHEA in order to improve the performance of the system and develop engineering options for future consideration.

\section{Computational Fluid Dynamics (CFD) Modelling and Simulation of NHEA}

The fluid dynamics analysis of the NHEA system only focuses on the thermodynamic interactions of the air and broken rock. In this section, the computational fluid dynamics (CFD) modeling of the NHEA system is presented in detail and the results of the simulations are discussed.

\subsection{Model Description}

\subsubsection{Geometry}

With reference to the actual geometry of the NHEA and the size and location of the 96 trench doors, a threedimensional model of the NHEA was built utilizing Ansys 17.1 - DesignModeler [4]. The geometry was discretized with $1,831,416$ tetrahedron mesh elements after mesh independence tests. The meshed geometry of the NHEA is presented in Fig. 3 and groups of trench doors (blocks) can be distinguished as the zones with higher mesh element density. The 
location and number of trench doors for Block 3 and 4 are rough estimates from the old prints of the mine. The dimensions of the NHEA are $698 \mathrm{~m}$ (x-direction), $694 \mathrm{~m}$ (y-direction), and $333 \mathrm{~m}$ (z-direction). The total volume of the model is 49 million $\mathrm{m} 3$ and the top surface area of the NHEA is $336,000 \mathrm{~m} 2$.

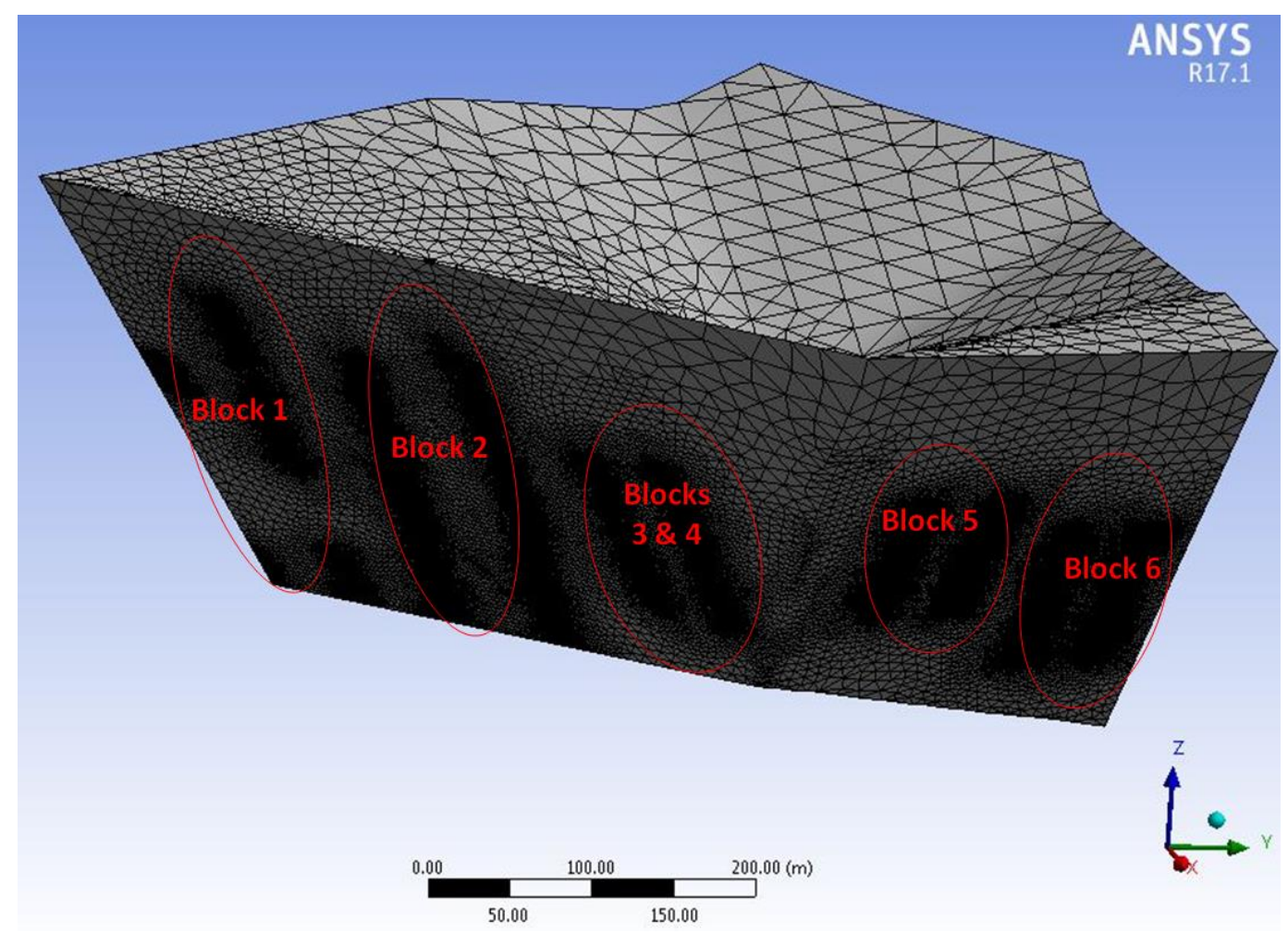

Fig. 3: The Natural Heat Exchange Area (NHEA) and trench doors with reference to the actual NHEA geometry. The geometry is built and meshed with Ansys 17.1 - DesignModeler [4]. Each group of trench doors is highlighted with red circles and the block numbers are labeled.

\subsubsection{Boundary Conditions}

The model considers transient boundary conditions for the transient heat transfer between ambient air and the broken rock with local thermal equilibrium (LTE). The inlet boundary condition is applied to the concave surface of the NHEA surface pit, which is exposed to the environment and is defined with Inlet-Velocity [4] at a constant airflow of $0.0024 \mathrm{~m} / \mathrm{s}$ (approximately $800 \mathrm{~m}^{3} / \mathrm{s}$ ) and a transient daily average ambient temperature as recorded by Environment Canada [5]. The transient temperature was set with User-Defined Function (UDF) written in the C language. The outlet boundary conditions are applied to all of the trench doors for each day and are defined with an Outflow of 0 if the door is closed and 1 if the door is open utilizing a Journal file [4]. The transient boundary conditions are defined for a period of 10 years using data from January 2008 to December 2015, repeating 2008 and 2009 data twice. It should be mentioned that the specified boundary conditions are limited to the availability and accuracy of the historical data recorded at the NHEA.

The volume of the broken rock in the NHEA is defined as a porous zone with a porosity of 0.2 based on the estimated bulking factor of 25 to $30 \%$ from the previous mining activities at the site [6]. The porous zone resistance (inverse of the porous zone permeability) is defined to be $75 \mathrm{~m}^{-2}$ based on the measured values of airflow and pressure at different locations within the NHEA. The porous zone is assumed to be initially homogenous, which might be a subject of change in further development of the CFD model. Table 1 reports the thermo-physical properties of the material content of the porous zone. 
Table 1: Thermo-physical properties of the model.

\begin{tabular}{|c|c|c|}
\cline { 2 - 3 } \multicolumn{1}{c|}{} & Property & Value \\
\hline \multirow{4}{*}{ Rock } & thermal conductivity & $2 \mathrm{~W} / \mathrm{m} . \mathrm{K}$ \\
\cline { 2 - 3 } & specific heat capacity & $800 \mathrm{~J} / \mathrm{Kg} . \mathrm{K}$ \\
\cline { 2 - 3 } & density & $3000 \mathrm{Kg} / \mathrm{m}^{3}$ \\
\cline { 2 - 3 } & permeability of porous zone & $0.0133 \mathrm{~m}^{2}$ \\
\cline { 2 - 3 } & porosity & $20 \%$ \\
\hline \multirow{3}{*}{ Air } & thermal conductivity & $0.0242 \mathrm{~W} / \mathrm{m} . \mathrm{K}$ \\
\cline { 2 - 3 } & specific heat capacity & $1006.43 \mathrm{~J} / \mathrm{Kg} . \mathrm{K}$ \\
\cline { 2 - 3 } & density & $1.205 \mathrm{Kg} / \mathrm{m}^{3}$ \\
\hline
\end{tabular}

\subsection{Numerical Methodology}

The model was solved by Ansys 17.1 - FLUENT using the Semi-Implicit Pressure Linked Equations (SIMPLE) algorithm, second-order upwind discretization and the Algebraic Multi-Grid (AMG) method [4]. The simulations ran for a period of 10 years with 3,653 time steps, 20 iterations per time step and a 86,400 seconds step-size (one day). The 10 year period of the simulation is defined with trial and error to assure that the simulation results are not affected by the arbitrary initialization of the model. Each simulation took 7 days to run on a 64-bit PC running Windows 7, with 32 GB RAM and an Intel®Core ${ }^{\mathrm{TM}} \mathrm{i} 7-4770 \mathrm{k} \mathrm{CPU} @ 3.5 \mathrm{GHz}$ processor.

\subsection{Results: Validation and Discussions}

In this work, the simulation results are validated based on the field-measured temperatures at each of the 96 trench doors and the ambient air temperature, for the year 2015. The measured temperatures at the trench doors as well as the ambient air temperature follow a sinusoidal wave-like behaviour year-round. The trench doors' temperature curves and the ambient temperature curves have the same frequency but different phase and amplitude. In this regard, each temperature curve has been characterised with a value of phase and temperature range. The validation of the simulated temperatures at the outlets for the year 2015 is initiated by comparing the characteristics of the simulated temperature curves (phase-shift from ambient air temperature curve, and the temperature range characteristics) at each outlet with measured temperature curves at the corresponding trench door. It is found that with the defined thermo-physical properties and boundary conditions, the model results are in good agreement with the field measurements. The temperature range at all the outlets matches with the temperature range at their corresponding trench doors. However, the phase-shift match between simulated and measured temperature curves is achieved at only 70 out of 96 trench doors. This might be due to inaccurate data for trench door locations, in addition to simplified boundary conditions and porous zone properties. Despite these, the current CFD model is capable of accounting for the fundamental thermodynamics behaviour of the NHEA.

Fig. 4 presents an abstract demonstration of the CFD simulation results. The three graphs in Fig. 5 compare the simulated and the measured temperature for the year 2015 (year 10 of the simulation) at three outlet locations: close to the surface, mid-depth of the NHEA and bottom of the NHEA. The location of each graph is indicated with a red arrow in the NHEA geometry presented in Fig. 6. The simulated temperature at the NHEA model outlets (in black) show an acceptable match with the measured temperature at the corresponding trench doors (in red), both in terms of temperature range and the shift from the ambient air temperature on the surface (in blue). The shift between the ambient air temperature graph and the trench door temperature graph, as well as the reduction in the temperature range is confirmation of the heat-exchange that occurs between rock and air in the porous zone. 


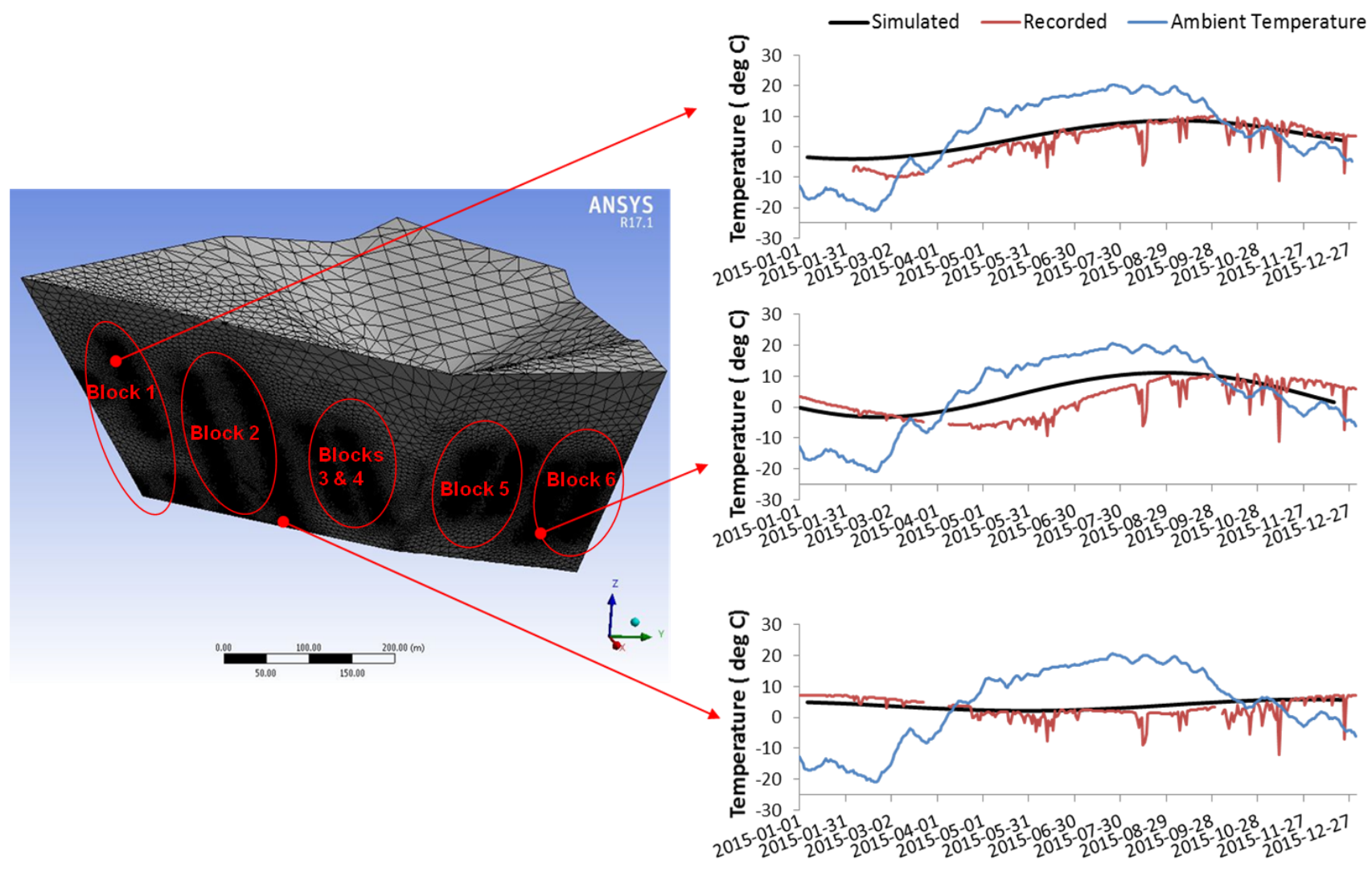

Fig. 4: Validation of the simulation results: three graphs compare the simulation result and the measured data for selected NHEA outlets in the final year of simulation (2015). The simulated temperature at the NHEA model outlets are in black, the measured temperature at the corresponding trench doors are in red and the ambient air temperature on the surface is in blue.

Fig. 5 presents the temperature contours in a cross section of the NHEA and airflow trajectories across the entire NHEA for December 2015. As the air travels deeper, with longer trajectories, the air temperature at the trench doors and within the porous zone moderates to a relatively constant temperature (approximately $4^{\circ} \mathrm{C}$ ) year-round. In contrast, closer to the surface, temperature follows a wave-like behaviour and is closer to the ambient air behaviour. This can be a measure for evaluation of engineering options with a calibrated CFD model.
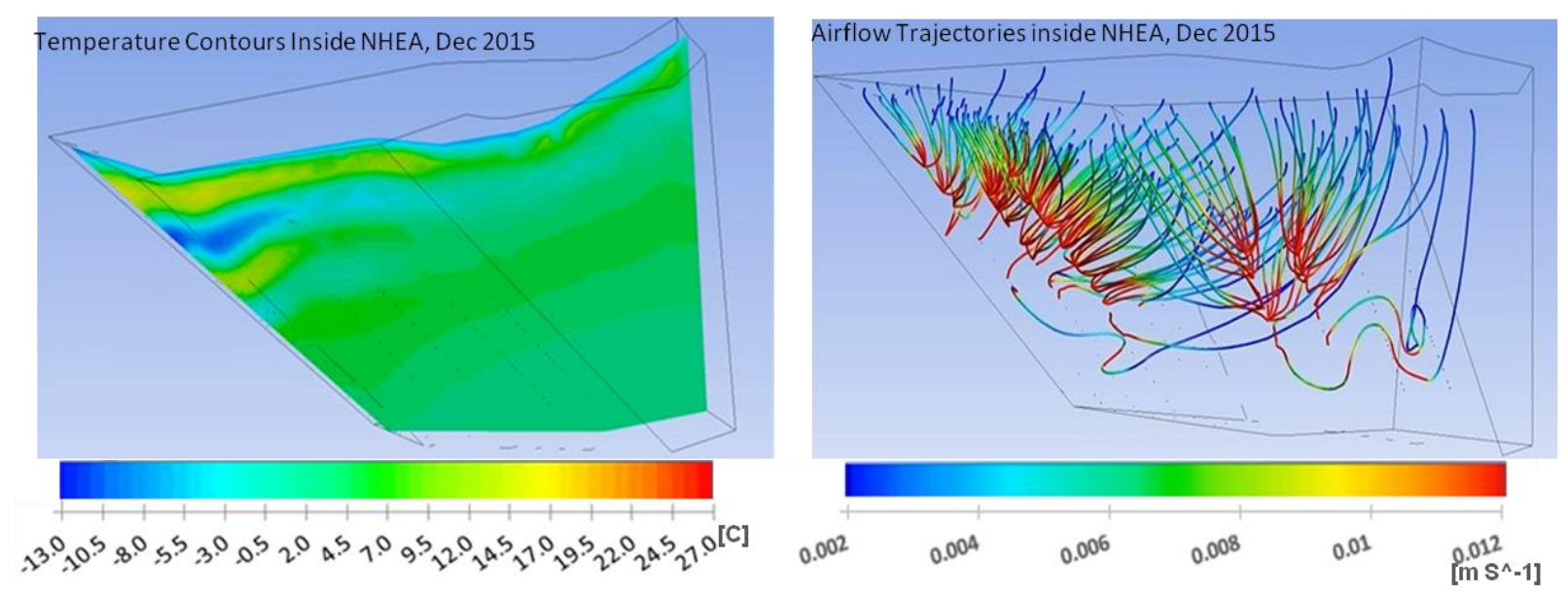

Fig. 5: Temperature contours in a cross section of the NHEA and airflow trajectories across the entire area for December 2015, produced with Ansys 17.1 - Post-Processing [4]. 


\section{Opportunities to Improve the NHEA's performance}

According to the qualitative analysis of the NHEA presented in Fig. 4, temperature variations at deeper parts of the NHEA are almost negligible and are typically steady. This can be attributed to having fewer trench doors in the bottom of the NHEA. Trench doors are the outlets of the system and the target for the path of least resistance for the airflow. A study of the old Creighton open-pit mine design maps can guide the selection of potential locations in the bottom of the pit that could connect to the underground ventilation system to pull more air from the bottom of the surface pit. This could be beneficial in the winter season because pulling more air from the bottom of the system is expected to promote the propagation of the mass of cold temperature to deeper parts of the NHEA. This could potentially provide larger cooling capacity during the summer months.

The airflow survey conducted at the NHEA trench doors and on the 800 Level collecting area has confirmed that there is a great amount of leakage in the system, which results in uncontrolled air entering the 800 Level collecting area. It is expected that locating and sealing the sources of the leakage in will provide the ability to lower the average temperature of the air on the collecting area ( 800 Level) for delivery to the deeper levels of the mine.

\section{Future Work}

The current computational fluid dynamics CFD model is capable of reproducing characteristics of the NHEA qualitatively, which leads to a better understanding of the system. A quantitative calibrated model is essential for the confident evaluation of engineering options to improve NHEA performance and provide cooler air to deeper levels of Creighton Mine. This can be achieved by manipulating the thermo-physical properties of the CFD model and replacing simplified assumptions by more realistic representations.

The next step towards a fully calibrated CFD model is to account for an inhomogeneous porous zone for the NHEA mass of broken rock volume. Determining the porosity and resistance parameters at different locations of the porous zone requires a very detailed airflow and pressure survey at the NHEA, which is now being conducted. Such a survey should also be accompanied by a search of the historical mining techniques that created the open-pit area at Creighton Mine. The calibrated model can provide guidance in the assessment of the expected performance of the NHEA after changes are applied, and in determining whether enough cooling capacity can be achieved.

\section{Conclusions and Summary}

The NHEA at Vale's Creighton Mine in Sudbury, Ontario, Canada is a valuable asset which provides natural cooling and heating for Creighton Mine's ventilating air. This system is being studied for performance improvements to increase its cooling capacity as deeper levels of the mine come into operation. The physics and thermal behaviour of the NHEA are described in this paper. In addition, a CFD model of the NHEA system for qualitative analysis was presented. Further field tests for supplementary knowledge of dynamic thermal behaviour of the system are being undertaken. The calibrated CFD model and historical data recorded at the NHEA will provide the principal guidance for potential engineered modifications to the system to enhance the NHEA.

\section{Acknowledgements}

The authors would like to acknowledge the support of Vale Canada, Ontario Division, including Paul Aho, Ventilation Specialist, and Brian Keen, Ventilation Supervisor, both of Creighton Mine and the financial support of the Ultra Deep Mining Network (UDMN) at the Centre for Excellence in Mining Innovation (CEMI). Contributing MIRARCO staff are gratefully acknowledged, primarily Alberto Romero.

\section{References}

[1] L. Fava, D. Millar, B. Anderson, S. Schafrik, D. O'Connor, C. Allen, "Modeling of the Natural Heat Exchange Area at Creighton Mine for operational decision support," 14th U.S./North American Mine Ventilation Symposium, Salt Lake City, 2012.

[2] R. Ramsden, C. Allen, D. Millar and T. Guse, "The use of natural cooling to delay and reduce refrigeration requirements," in The Mine Ventilation Society of South Africa, Sun City, 2014.

[3] M. J.-G. Sylvestre, "Heating and ventilation study of Inco's Creighton Mine," M.S. thesis: Department of Mining and Metallurgical Engineering, McGill University, Canada, 1999. 
[4] I. ANSYS, "Ansys 17.1," [Online]. Available: www.ansys.com

[5] Environment Canada, "Government of Canada," Canada, [Online]. Available: http://climate.weather.gc.ca/climate_data/daily_data_e.html?StationID=50840

[6] S. Schafrik, "The use of packed sphere modelling for airflow and heat exchange analysis in broken or fragmented rock," Ph.D. Thesis: Faculty of Graduate Studies Laurentian University, Canada, 2014. 